\title{
On the Logical Inconsistency of the Special Theory of Relativity
}

\section{Stephen John Crothers}

Theoretical Physics Unit, Institute for Basic Research, Palm Harbor, USA

\section{Email address:}

thenarmis@yahoo.com

\section{To cite this article:}

Stephen John Crothers. On the Logical Inconsistency of the Special Theory of Relativity. American Journal of Modern Physics. Vol. 6, No. 3, 2017, pp. 43-48. doi: 10.11648/j.ajmp.20170603.12

Received: February 22, 2017; Accepted: April 14, 2017; Published: June 13, 2017

\begin{abstract}
Einstein's Special Theory of Relativity requires systems of clock-synchronised stationary observers and the Lorentz Transformation. Without both, the Theory of Relativity fails. A system of clock-synchronised stationary observers is proven inconsistent with the Lorentz Transformation, because it is Galilean. The Special Theory of Relativity insists that Galilean systems must transform not by the Galilean Transformation, but by the non-Galilean Lorentz Transformation. The Theory of Relativity is therefore invalid due to an intrinsic logical contradiction.
\end{abstract}

Keywords: Special Relativity, Simultaneity, Stationary Observers, Clock-Synchronised Observers, Lorentz Transformation, Time

\section{Introduction}

It has recently been proven by Engelhardt [1] that Einstein's method of synchronising clocks in his Special Theory of Relativity is inconsistent with the Lorentz Transformation. This inconsistency is in fact due to an inherent logical contradiction in Special Relativity, because Einstein incorrectly assumed that systems of clocksynchronised stationary observers are consistent with the Lorentz Transformation.

"Now, however, as we know how to judge whether two, or more, clocks show the same time simultaneously and run in the same way, we can very well imagine as many clocks as we like in a given CS. The clocks are all at rest relative to the CS. They are 'good' clocks and are synchronised, which means that they show the same time simultaneously." [2 \$3]

"We have so far defined only an 'A time' and a 'B time.' We have not defined a common 'time' for $A$ and $B$, for the latter cannot be defined at all unless we establish by definition that the 'time' required by light to travel from $A$ to $B$ equals the 'time' it requires to travel from $B$ to $A$. Let a ray of light start at the direction of $A$, and arrive again at $A$ at the 'A time' $t^{\prime}$ '."

"In accordance with definition the two clocks synchronize if

$$
t_{B}-t_{A}=t_{A}^{\prime}-t_{B} . ”[3, \S 3]
$$

"We envisage a rigid sphere of radius $R$, at rest relatively to the moving system $k$, and with its centre at the origin of coordinates of $k$.... A rigid body which, measured in a state of rest, has the form of a sphere, therefore has in a state of motion - viewed from the stationary system - the form of an ellipsoid of revolution with the axes

$$
R \sqrt{1-v^{2} / c^{2}}, R, R . ”
$$

"Thus, whereas the $Y$ and $Z$ dimensions of the sphere (and therefore of every rigid body of no matter what form) do not appear modified by the motion, the $X$ dimension appears shortened in the ratio 1: $\sqrt{ }\left(1-v^{2} / c^{2}\right)$, i.e. the greater the value of $v$, the greater the shortening." $[3, \S 4]$

"Therefore,

$$
\tau=t \sqrt{1-v^{2} / c^{2}}=t-\left(1-\sqrt{1-v^{2} / c^{2}}\right) t
$$

whence it follows that the time marked by the clock (viewed in the stationary system) is slow by $1-\sqrt{(}\left(1-v^{2} / c^{2}\right)$, seconds per second" [3, §4].

"Thus with the help of certain imaginary physical experiments we have settled what is to be understood by synchronous stationary clocks located at different places, and have evidently obtained a definition of 'simultaneous', or 
'synchronous', and of 'time'. The 'time' of an event is that which is given simultaneously with the event by a stationary clock located at the place of the event, this clock being synchronous, and indeed synchronous for all time determinations, with a specified stationary clock." [3, $\$ 1]$

In $\S 2$ I mathematically construct a system of stationary observers and apply the Lorentz Transformation to prove that no observer can be clock-synchronised. In $\S 5$ I mathematically construct a system of clock-synchronised observers and apply the Lorentz Transformation to prove that not all observers are stationary.

That systems of clock-synchronised stationary observers are logically inconsistent with the Lorentz Transformation entirely subverts the Theory of Relativity.

\section{Systems of Stationary Observers}

A system of clock-synchronised stationary observers is an essential feature of Special Relativity. Einstein [3, §3] holds that the Lorentz Transformation associates coordinates $x, y, z$, $t$ of his 'stationary system' $K$ with the coordinates $\xi, \eta, \zeta, \tau$ of his 'moving system' $k$. A system of clock-synchronised stationary observers and the Lorentz Transformation are the bases for Einstein's time dilation and length contraction. It is regarded in general by physicists $[4, \S 12.1]$ that a stationary system of observers $k$ which are clock-synchronised when at rest are not synchronised when they all move together with respect to a clock-synchronised 'stationary system' $K$, as illustrated in figure 1.

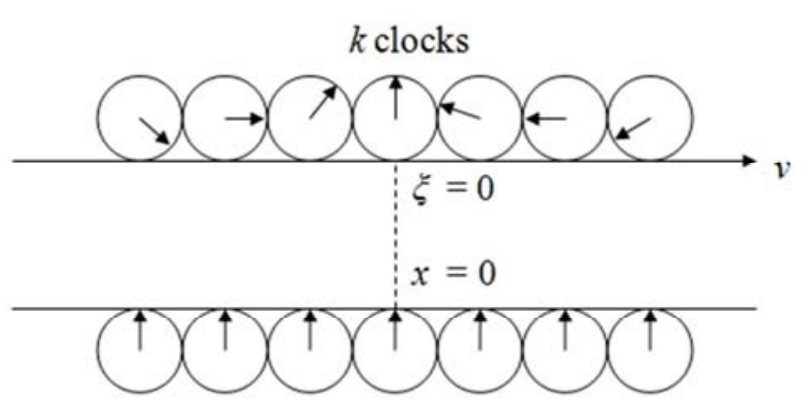

\section{$K$ clocks}

Figure 1. All the synchronised clocks in the 'stationary system' $K$ read the same time at all positions in the $K$ system. All the clocks in the 'moving system' $k$ do not read the same time according to the $K$ system, despite being synchronised with respect to the $k$ system. Only at $x=\xi=0$ do the clocks depicted read the same time in both systems, where $t=\tau=0$.

In figure 1 , clocks to the left of the central clock in the 'moving system' $k$ are 'ahead' of the central clock and those to the right of it 'lag' it, according to the 'stationary system' $K$ where all the clocks therein always read the same time $t$. After a time $t>0$ in $K$ the moving observers' clocks advance to the right and the hands on the moving clocks advance, but, according to any observer in $K$, they do not read the same time. As time $t$ increases all the hands of the 'stationary' clocks advance by the same amount and all observers' clocks in $K$ still read the common time $t$ - they are synchronised. However, for any $x$ and $t$ in the 'stationary system' $K$ there is always an observer located at someplace $\neq x$ with a clock that reads $\neq t$, thereby contradicting Einstein's assumption of synchronisation of clocks in his 'stationary system' $K$. Recall the Lorentz Transformation equations for the time $\tau$ at position $\xi$ in the 'moving system' $k$ according to the 'stationary system' $K$ :

$$
\begin{aligned}
& \tau=\beta\left(t-v x / c^{2}\right), \\
& \xi=\beta(x-v t), \\
& \eta=y, \\
& \varsigma=z, \\
& \beta=1 / \sqrt{1-v^{2} / c^{2}} .
\end{aligned}
$$

To ensure a system of stationary observers $K$ by mathematical construction, set,

$$
x_{\sigma}=\sigma x_{1}
$$

where $\sigma \in \mathfrak{R}$ denotes the observer, $x_{\sigma}$ the location of that observer and $x_{1} \neq 0$. All the observers $\sigma$ are stationary. All observers $\sigma$ have an Einstein clock, reading the time $t_{\sigma}$.

The time $t_{\sigma}$ associated with observer $\sigma$ located at $x_{\sigma}$ must now be quantified by means of the Lorentz Transformation. The equation for $\xi$ in (1) cannot be used for this purpose because it leads to a contradiction concerning the presence of arbitrarily many observers. Therefore only the equation for $\tau$ in (1) can be used. Therefore, set,

$$
t_{\sigma}-\frac{v x_{\sigma}}{c^{2}}=t_{\sigma}-\frac{v \sigma x_{1}}{c^{2}}=t_{1}-\frac{v x_{1}}{c^{2}}
$$

whence,

$$
t_{\sigma}=t_{1}+\frac{(\sigma-1) v x_{1}}{c^{2}} \text {. }
$$

Then, using the relation for $\xi$ of the Lorentz Transformation (1),

$$
\begin{array}{r}
\xi_{\sigma}=\beta\left(x_{\sigma}-v t_{\sigma}\right)=\beta\left[\left(\frac{\sigma}{\beta^{2}}+\frac{v^{2}}{c^{2}}\right) x_{1}-v t_{1}\right], \\
=\beta\left\{\left[\sigma\left(1-\frac{v^{2}}{c^{2}}\right)+\frac{v^{2}}{c^{2}}\right] x_{1}-v t_{1}\right\},
\end{array}
$$

leading to some sample values in table 1.

Table 1. Sample values of observer location and associated time according to the stationary system $K$.

\begin{tabular}{lllll}
\hline $\boldsymbol{\sigma}$ & $\mathbf{x}_{\boldsymbol{\sigma}}$ & $\mathbf{t}_{\boldsymbol{\sigma}}$ & $\boldsymbol{\xi}_{\boldsymbol{\sigma}}$ & $\boldsymbol{\tau}_{\boldsymbol{\sigma}}$ \\
\hline-1 & $-\mathrm{x}_{1}$ & $t_{1}-\frac{2 v x_{1}}{c^{2}}$ & $\beta\left[\frac{\left(2 v^{2}-c^{2}\right)}{c^{2}} x_{1}-v t_{1}\right]$ & $\beta\left(t_{1}-\frac{v x_{1}}{c^{2}}\right)$ \\
\hline
\end{tabular}




\begin{tabular}{lllll}
\hline $\boldsymbol{\sigma}$ & $\mathbf{x}_{\boldsymbol{\sigma}}$ & $\mathbf{t}_{\boldsymbol{\sigma}}$ & $\xi_{\boldsymbol{\sigma}}$ & $\boldsymbol{\tau}_{\boldsymbol{\sigma}}$ \\
\hline 0 & 0 & $t_{1}-\frac{v x_{1}}{c^{2}}$ & $\beta\left[\frac{v^{2}}{c^{2}} x_{1}-v t_{1}\right]$ & $\beta\left(t_{1}-\frac{v x_{1}}{c^{2}}\right)$ \\
$1 / 2$ & $\frac{x_{1}}{2}$ & $t_{1}-\frac{v x_{1}}{2 c^{2}}$ & $\beta\left[\frac{\left(c^{2}+v^{2}\right)}{2 c^{2}} x_{1}-v t_{1}\right]$ & $\beta\left(t_{1}-\frac{v x_{1}}{c^{2}}\right)$ \\
1 & $\mathrm{x}_{1}$ & $t_{1}$ & $\beta\left(x_{1}-v t_{1}\right)$ & $\beta\left(t_{1}-\frac{v x_{1}}{c^{2}}\right)$ \\
2 & $2 \mathrm{x}_{1}$ & $t_{1}+\frac{v x_{1}}{c^{2}}$ & $\beta\left[\frac{\left(2 c^{2}-v^{2}\right)}{c^{2}} x_{1}-v t_{1}\right]$ & $\beta\left(t_{1}-\frac{v x_{1}}{c^{2}}\right)$ \\
3 & $3 \mathrm{x}_{1}$ & $t_{1}+\frac{2 v x_{1}}{c^{2}}$ & $\beta\left[\frac{\left(3 c^{2}-2 v^{2}\right)}{c^{2}} x_{1}-v t_{1}\right]$ & $\beta\left(t_{1}-\frac{v x_{1}}{c^{2}}\right)$ \\
& & &
\end{tabular}

The Stationary Lorentz Transformation is,

$$
\begin{aligned}
& \tau=\beta\left(t_{\sigma}-v x_{\sigma} / c^{2}\right) \\
& x_{\sigma}=\sigma x_{1} \\
& \xi_{\sigma}=\beta\left(x_{\sigma}-v t_{\sigma}\right)=\beta\left[\left(\frac{\sigma}{\beta^{2}}+\frac{v^{2}}{c^{2}}\right) x_{1}-v t_{1}\right] \\
& t_{\sigma}=t_{1}+\frac{(\sigma-1) v x_{1}}{c^{2}} \\
& \eta=y \\
& \varsigma=z \\
& \beta=1 / \sqrt{1-v^{2} / c^{2}} \\
& \sigma \in \Re .
\end{aligned}
$$

From table 1 , for any time $t_{1} \geq 0$ of the 'stationary system' $K$ there are any number of locations $x_{\sigma} \neq x_{1}$ at which there is a stationary observer $\sigma$ whose clock reading is $t_{\sigma} \neq t_{1}$, thereby contradicting Einstein's assumption of clock synchronisation for all observers in the stationary system $K$. Moreover, every stationary observer $\sigma$ in $K$ observes the same time $\tau$ on the clocks in $k$. This is consistent with two different interpretations, both of which are opposite to those of Einstein:

(a) According to every observer in the stationary system of observers $K$, all the clocks in the system $k$ are synchronised but not stationary, because system $k$ is moving, and all the clocks in the system $K$ are stationary but not synchronised.

(b) Every stationary observer $\sigma$ at position $x_{\sigma}$ in the system $K$ observes the same clock in $k$, finding that it indicates the same time $\tau$ for them all, but also find that the observed clock is at a different location $\xi_{\sigma}$ in $k$.

Either way, Einstein's system of clock-synchronised stationary observers is inconsistent with the Lorentz Transformation.

Suppose an event occurs in the stationary system of observers $K$ at $x_{1}$ at associated time $t_{1}$. Then according to all other observers $x_{\sigma}$ in $K$ the event occurs at $t_{\sigma} \neq t_{1}$. No events in $K$ can be simultaneous according to any of its observers $\sigma$. According to all the observers of system $K$, all the observers in the moving system see the event in $K$ simultaneously. This is revealed mathematically by the Inverse Stationary Lorentz Transformation:

$$
\begin{aligned}
& t=\beta\left(\tau_{\sigma}+v \xi_{\sigma} / c^{2}\right), \\
& \xi_{\sigma}=\sigma \xi_{1} \\
& x_{\sigma}=\beta\left(\xi_{\sigma}+v \tau_{\sigma}\right)=\beta\left[\left(\frac{\sigma}{\beta^{2}}+\frac{v^{2}}{c^{2}}\right) \xi_{1}+v \tau_{1}\right], \\
& \tau_{\sigma}=\tau_{1}-\frac{(\sigma-1) v \xi_{1}}{c^{2}} \\
& y=\eta \\
& z=\varsigma \\
& \beta=1 / \sqrt{1-v^{2} / c^{2}} \\
& \sigma \in \Re .
\end{aligned}
$$

None of the stationary observers $\sigma$ in $k$ can be clocksynchronised and all see the same time $t$ at all observer locations in the moving system $K$ for any event at $\xi_{\sigma}$ at time $\tau_{\sigma}$ in $k$, which cannot be simultaneous for any observer in $k$.

For a system of stationary observers, relativity of simultaneity is opposite to that advanced by Einstein from his Special Theory of Relativity. His relativity of simultaneity obtains effectively from his unwitting restriction of observers to just one observer, $\sigma=1$ in table 1, in his 'stationary system' $K$, and letting it speak for all observers in $K$, due to his false assumption that a system of clock-synchronised stationary observers is consistent with the Lorentz Transformation. Setting $\sigma=1$ in (6), the Lorentz Transformation used by Einstein is recovered. A system $S$ that contains only one observer cannot, by its singular character, synchronise its clock with anything or judge 'simultaneous' events in $S$.

Physicists since Einstein have only ever invoked the case of observer $\sigma=1$ in table 1 ; in other words they have only ever considered this one observer in the system $K$, on the incorrect assumption that any desired system of observers can be a system of clock-synchronised stationary observers consistent with the Lorentz Transformation. From this incorrect assumption they conclude that the conditions and effects are the same for all observers in the system $K$; hence a common time dilation and length contraction relative to any and all observers of a moving system. Table 1 however reveals that this is not the case.

\section{3. 'Length Contraction' for a System of Stationary Observers}

At any instant of time in the stationary system $K$ described by equations (2) - (7), let a rigid rod in the moving system $k$ have a length $\Delta \xi=l_{0}$ when at rest relative to the moving system $k$. From (6), 


$$
\Delta \xi=l_{0}=\xi_{\sigma}-\xi_{\rho}=\frac{(\sigma-\rho) x_{1}}{\beta}=\frac{\Delta x}{\beta} .
$$

Therefore,

$$
\Delta x=\beta l_{0}=\frac{l_{0}}{\sqrt{1-\frac{v^{2}}{c^{2}}}} .
$$

Hence, all the observers $x_{\sigma}$ of the stationary system $K$ observe not length contraction, but length extension.

\section{Time Dilation for a System of Stationary Observers}

By means of the Inverse Stationary Lorentz Transformation (7),

$$
\Delta \tau_{\sigma}=\Delta \tau_{1}=\frac{\Delta t}{\beta}=\Delta t \sqrt{1-\frac{v^{2}}{c^{2}}} .
$$

Thus, although no observer in the stationary system $K$ is clock-synchronised, every observer $x_{\sigma}$ of the stationary system $K$ observes the same time interval in $K$ and the same time-dilated interval in $k$, but at the expense of length contraction and of clock-synchronised stationary observers. This is irreconcilable with Einstein's theory.

\section{Systems of Clock-Synchronised \\ Observers}

The locations of the observers $\sigma$ associated with the common time $t_{\sigma}=t$ for all values of $\sigma$ must now be quantified by means of the Lorentz Transformation. The equation for $\xi$ in (1) cannot be used for this purpose because it leads to a contradiction concerning the presence of arbitrarily many observers. Therefore only the equation for $\tau$ in (1) can be used. Therefore, set,

$$
\begin{aligned}
& t=\frac{\tau_{1}}{\beta}+\frac{v x_{1}}{c^{2}}=\frac{\tau_{\sigma}}{\beta}+\frac{v x_{\sigma}}{c^{2}}, \\
& \tau_{\sigma}=\sigma \tau_{1},
\end{aligned}
$$

where $\sigma$ labels an observer and $x_{\sigma}$ is the location of that observer in the clock-synchronised system $K$. Thus all $x_{\sigma}$ read the same time $t$. Solving (10) for $x_{\sigma}$ gives,

$$
x_{\sigma}=\frac{(1-\sigma) c^{2} \tau_{1}}{v \beta}+x_{1} .
$$

From (10),

$$
\tau_{1}=\beta\left(t-\frac{v x_{1}}{c^{2}}\right)
$$

Putting (12) into (11) gives,

$$
x_{\sigma}=\frac{(1-\sigma) c^{2} t}{v}+\sigma x_{1} .
$$

Although all observers in $K$ are clock-synchronised by (10) to a common time $t$, only $x_{1}$ is not a function of the time $t$. Thus, only $x_{1}$ is stationary. All other observers in $K$ cannot be stationary. The Clock-Synchronised Lorentz Transformation is,

$$
\begin{aligned}
& \tau_{\sigma}=\beta\left(t-v x_{\sigma} / c^{2}\right)=\sigma \tau_{1}, \\
& \xi_{\sigma}=\beta\left(x_{\sigma}-v t\right), \\
& x_{\sigma}=\frac{(1-\sigma) c^{2} t}{v}+\sigma x_{1}, \\
& \eta=y, \\
& \varsigma=z, \\
& \beta=1 / \sqrt{1-v^{2} / c^{2}},
\end{aligned}
$$

The Inverse Clock-Synchronised Lorentz Transformation is,

$$
\begin{aligned}
& t_{\sigma}=\beta\left(\tau+v \xi_{\sigma} / c^{2}\right)=\sigma t_{1}, \\
& x_{\sigma}=\beta\left(\xi_{\sigma}+v \tau\right), \\
& \xi_{\sigma}=\frac{(\sigma-1) c^{2} \tau}{v}+\sigma \xi_{1}, \\
& y=\eta \\
& z=\varsigma \\
& \beta=1 / \sqrt{1-v^{2} / c^{2}}
\end{aligned}
$$

Although all observers in $k$ are clock-synchronised by (15) to a common time $\tau$, only $\xi_{1}$ is not a function of the time $\tau$. Thus, only $\xi_{1}$ is stationary. All other observers in $k$ cannot be stationary.

From (14),

$$
\frac{d x_{\sigma}}{d t}=\frac{(1-\sigma) c^{2}}{v}<c,
$$

whence,

$$
1-\frac{v}{c}<\sigma
$$

From (15),

$$
\frac{d \xi_{\sigma}}{d \tau}=\frac{(\sigma-1) c^{2}}{v}<c
$$

whence,

$$
\sigma<1+\frac{v}{c}
$$


Combining (16) and (17),

$$
1-\frac{v}{c}<\sigma<1+\frac{v}{c}
$$

All observers in the clock-synchronised system $K$ observe that none of the clocks in system $k$ are synchronised. Conversely, all observers in the clock-synchronised system $k$ observe that none of the clocks in system $K$ are synchronised. Setting $\sigma=1$ in (14) recovers the Lorentz Transformation used by Einstein. It pertains to only one observer in system $K$ and one observer in system $k$. By assuming systems of clocksynchronised stationary observers consistent the Lorentz Transformation, Einstein incorrectly permitted the observer $\sigma$ $=1$ to speak for all observers.

\section{Length Contraction for a System of Clock-Synchronised Observers}

From (14), at any instant of time $t$,

$$
\begin{aligned}
\Delta \xi & =\xi_{\sigma}-\xi_{\rho}=\beta\left(x_{\sigma}-x_{\rho}\right) \\
& =\beta(\sigma-\rho) x_{1}=\beta \Delta x
\end{aligned}
$$

Therefore,

$$
\Delta x=\frac{\Delta \xi}{\beta}=\Delta \xi \sqrt{1-\frac{v^{2}}{c^{2}}} .
$$

This is Einstein's 'length contraction' equation. Clocksynchronised observers $x_{\sigma}$ and $x_{\rho}$ of the system $K$ see the same length contraction, at the expense of being stationary observers.

\section{7. 'Time Dilation' for a System of Clock-Synchronised Observers}

Since all observers are clock-synchronised with respect to their own systems, all observers in the $K$ system observe the common clock time-interval $\Delta t$. Observer $x_{\sigma}$ of system $K$ watches the clock of $\xi_{\sigma}$ in the 'moving system' $k$ and observes the clock time-interval $\Delta \tau_{\sigma}$ of $\xi_{\sigma}$ in system $k$. Then from (14),

$$
\Delta \tau_{\sigma}=\sigma \beta \Delta t
$$

Thus, after a time interval $\Delta t$ in $K$ any observer $x_{\sigma}$ in the clock-synchronised system $K$ reads the time-interval $\Delta \tau_{\sigma}$ at $\xi_{\sigma}$ in the $k$ system. Each observer $x_{\sigma}$ observes a different time and a different time-interval on the corresponding clock held by observer $\xi_{\sigma}$ in system $k$. For example, the observer $\sigma=1$ located at $x_{1}$ in system $K$ observes not time dilation at $\xi_{1}$ but time expansion at $\xi_{1}: \Delta \tau_{1}=\beta \Delta t$. The observer $\sigma=1 / \beta$ located at $x_{1 / \beta}$ in system $K$ observes no change in the time-interval of the clock of $\xi_{1 / \beta}$ in system $k: \Delta \tau_{1 / \beta}=\Delta t$. The observer $\sigma=1 / \beta^{2}$ located at $x_{1 / \beta^{2}}$ observes the time interval $\Delta \tau_{1 / \beta^{2}}$ at $\xi_{1 / \beta^{2}}$ to be,

$$
\Delta \tau_{1 / \beta^{2}}=\frac{\Delta t}{\beta}=\Delta t \sqrt{1-\frac{v^{2}}{c^{2}}},
$$

which is Einstein's time dilation equation. Note that in all cases, $\Delta \tau_{\sigma}=\sigma \Delta \tau_{1}$, in accordance with (14), since $\Delta \tau_{1}=\beta \Delta t$. Conversely, all observers $\xi_{\sigma}$ in the $k$ system read a common time $\tau$ and common time interval $\Delta \tau$, finding that the clock at $x_{\sigma}$ in $K$ reads, from (15), the time interval,

$$
\Delta t_{\sigma}=\sigma \beta \Delta \tau
$$

\section{The Systems of Observers}

To facilitate comparison of observers, write (6) with capital letters,

$$
\begin{aligned}
& \tau=\beta\left(t_{\sigma}-v X_{\sigma} / c^{2}\right) \\
& X_{\sigma}=\sigma X_{1} \\
& \xi_{\sigma}=\beta\left(X_{\sigma}-v t_{\sigma}\right)=\beta\left[\left(\frac{\sigma}{\beta^{2}}+\frac{v^{2}}{c^{2}}\right) X_{1}-v t_{1}\right] \\
& t_{\sigma}=t_{1}+\frac{(\sigma-1) v X_{1}}{c^{2}} \\
& \eta=y \\
& \varsigma=z \\
& \beta=1 / \sqrt{1-v^{2} / c^{2}} \\
& \sigma \in \Re .
\end{aligned}
$$

Now set $\sigma=1$. The stationary observer $\sigma=1$ is located at $X_{1}$. This observer is not clock synchronised with any other observers in its stationary system $K$. Now set $\sigma=1$ in (14). The observer $x_{1}$, although stationary (the only stationary observer in its system), is clock synchronised with all other observers in its clock-synchronised system $K$. Moreover, the range on $\sigma$ in (22) is not the same as in (14), since in the latter the values of $\sigma$ are constrained by the speed $v$ and the speed of light $c$. Thus, observers located at $X_{1}$ and $x_{1}$ are not equivalent observers. Nonetheless, owing to his false assumption, Einstein makes them not only the same, but also the speaker for all other observers in his 'stationary' system $K$, effectively making each of his two systems $K$ and $k$, systems that contain only one observer in each.

To reaffirm that only the observer $\sigma=1$ is contained in Einstein's theory, set the position $x_{\sigma}$ in the stationary system (6) equal to the position $x_{\sigma}$ of the clock-synchronised system (14),

$$
\sigma x_{1}=\frac{(1-\sigma) c^{2} t}{v}+\sigma x_{1} .
$$

Thus the only observer is $\sigma=1$ for each system.

Similarly, set the time for the stationary system (6) equal to the time for the clock-synchronised system $\left(t=t_{l}\right.$ in the 
latter since its clocks are all synchronised),

$$
t_{\sigma}=t_{1}+\frac{(\sigma-1) v x_{1}}{c^{2}}=t_{1}=t .
$$

Thus, once again only the observer $\sigma=1$ is present.

\section{Conclusions}

By his clock synchronisation method Einstein attempted to ensure that time at all places within a given stationary system of observers is the same, despite subsequently invoking the Lorentz Transformation. Yet clock-synchronised stationary systems of observers are inconsistent with the Lorentz Transformation. Special Relativity is thereby invalid due to an insurmountable logical contradiction. Systems of clocksynchronised stationary observers are Galilean.

Einstein $[3, \S 1]$ defined time by means of his clocks. However, time is no more defined by a clock than pressure is defined by a pressure gauge, speed by a speedometer, heat by a thermometer, or gravity by a spring. Measuring instruments are invented to measure something other than themselves. Einstein's clocks measure only themselves. By defining 'time' by his clocks, Einstein detached time from physical reality. Nonetheless, all textbook writers on the subject reiterate Einstein's false assumptions, for example [4-18]. They all suffer, necessarily, from the same logical inconsistency as Einstein's 1905 paper.

\section{References}

[1] W. Engelhardt, Physics Essays, 29, 4, 2016

[2] S. J. Crothers, Einstein's Anomalous Clock Synchronisation, http://vixra.org/pdf/1702.0214v1.pdf
[3] A. Einstein, On the electrodynamics of moving bodies, Ann. Phys, 17, 1905

[4] D. J. Griffiths, Introduction to Electrodynamics, 4th Ed., PearsonEducation Inc., 2013

[5] A. Einstein, The Meaning of Relativity, Princeton University Press, 1988

[6] A. Einstein, Relativity the Special and the General Theory, Methuen, London, 1954

[7] E. F. Taylor, J. A. Wheeler, Spacetime Physics, 2nd ed., W. H. Freeman and Company, New York, 1992

[8] R. d'Inverno, Introducing Einstein's Relativity, Oxford University Press, 1992

[9] B. W. Carroll, D. A. Ostlie, Modern Astrophysics, Addison Wesley Longman, 1996

[10] R. A. Mould, Basic Relativity, Springer, 1994

[11] R. C. Tolman, Relativity Thermodynamics and Cosmology, Dover Publications Inc., New York, 1987

[12] S. H. Radin, R. T. Folk, Physics for Scientists and Engineers, Prentice Hall Inc., 1982

[13] D. McMahon, Relativity Demystified, McGraw-Hill, 2006

[14] B. Schutz, A First Course in General Relativity, Cambridge University Press, 2009

[15] A. Shadowitz, Special Relativity, Dover Publications Inc., 1988

[16] W. Rindler, Introduction to Special Relativity, Clarendon Press, 1982

[17] W. Pauli, The Theory of Relativity, Dover Publications Inc., New York, 1981

[18] R. E. Turner, Relativity Physics, Routledge \& Kegan Paul, 1984 